\title{
ARTIGOS
}

\section{EMPREGO DA ASSOCIAÇÃO TETRACICLINA E QUININO NO TRATAMENTO DA MALÁRIA CAUSADA PELO PLASMODIUM FALCIPARUM}

\author{
L.C.B. Barata, M. Boulos e A.P. Dutra*
}

\begin{abstract}
Cento e dez pacientes com malária falciparum não complicada, provenientes da Amazônia brasileira, foram tratados com cloridrato de tetraciclina, na dose de $250 \mathrm{mg}$ a cada 6 horas por 7 dias, associado ao sulfato de quinino na dose de 1,5 a 2,0 gramas/dia nos 3 ou 4 dias iniciais de tratamento. Os pacientes tiveram acompanhamento clínico e parasitológico por pelo menos 7 dias e em todos observou-se a negativação da parasitemia após o tratamento. Setenta e cinco pacientes foram acompanhados por 28 dias ou mais e destes 71 tiveram resposta do tipo $S$ e 4 do tipo RI. $O$ cloridrato de tetraciclina associado ao sulfato de quinino mostrou-se altamente eficaz no tratamento de malária falciparum não complicada, sendo bem tolerado e portanto útil na terapêutica das infecçóes por $\mathbf{P}$. falciparum multirresistentes contraídas na Amazônia.
\end{abstract}

Palavras chaves: Quimioterapia. Quinino + tetraciclina. Malária. P. falciparum.

A elevada incidência na Amazônia brasileira de infecçōes por $P$. falciparum resistentes à cloroquina $\mathrm{e}$ também, mais recentemente, à associação sulfadoxinapirimetamina, traz preocupações justificadas no que diz respeito ao tratamento e ao controle da malária no Brasil.

Desde a descrição, no inicio da década de 60 , dos primeiros casos de malária falciparum resistentes à cloroquina progressivamente o problema vem se agravando em nosso país ${ }^{5}$. Não só a resistência à cloroquina se disseminou por toda a Amazónia, como também passou a ser encontrada resistência a outros esquemas terapêuticos alternativos ${ }^{18}$. Um estudo por nós realizado no ano de 1983 revelou a virtual ineficácia da combinação sulfadoxina-pirimetamina no tratamento de infecções por $P$. falciparum contraidas nas regiōes do norte de Mato Grosso, sudoeste do Pará e no Estado de Rondônia ${ }^{2}$.

Esta grave situação justifica a necessidade de se estudar regimes terapêuticos novos a fim de se enfrentar o desafio representado pelas infecções por $P$. falciparum multirresistentes.

O presente estudo objetivou avaliar a eficácia do uso de tetraciclina combinada ao quinino no tratamento de pacientes com malária falciparum contraidas na Amazônia brasileira.

\section{CASUÍSTICA E MÉTODOS}

O estudo foi conduzido pela SUCEN na cidade

* SUCEN - Superintendência de Controle de Endemias, São Paulo, SP.

Recebido para publicação em 29/5/1985. de São Paulo, com pacientes acometidos por malária falciparum adquirida na Amazônia. Para ser incluído no estudo o paciente deveria ter mais de 7 anos de idade, parasitemia inferior a 100.000 formas assexuadas por $\mathrm{mm}^{3} \mathrm{e}$ não apresentar nenhum indício de complicações decorrentes da infecção.

Os pacientes foram tratados ambulatorialmente e permaneceram em área sem transmissão de malária durante todo período de acompanhamento. Os controles parasitários foram programados para serem efetuados nos dias $0,3,7,14,21$ e 28 (considerandose o dia 0 como o de início do tratamento). Quando possivel foi realizado controle parasitário também após o dia 28. Ao retornarem para realização dos controles parasitários os pacientes eram indagados quanto ao uso correto da medicação e à ocorrência de possiveis efeitos colaterais. Cento e trinta e quatro pacientes receberam tratamento com tetraciclina, mas 24 deles não retornaram para controle parasitário ou só o fizeram uma única vez antes do dia 7 e, dessa forma, foram excluídos do estudo.

Dos 110 pacientes que fizeram parte do estudo, 98 (89\%) eram do sexo masculino. A idade variou de 8 a 68 anos sendo que 94 (85\%) tinham entre 15 e 44 anos.

O local provável de infecção dos pacientes foi o seguinte: 42 (38\%) em Rondônia, 33 (30\%) em Mato Grosso, 30 (27\%) no Pará e $5(4,5 \%)$ em outros estados da Amazônia.

A parasitemia variou de 120 a 87.120 formas assexuadas $/ \mathrm{mm}^{3}$, sendo em média $8866 / \mathrm{mm}^{3}$.

A todos os pacientes foi fornecido cloridrato de tetraciclina por 7 dias na dose de $250 \mathrm{mg}$ a cada 6 
Barata LCB, Boulos M, Dutra AP. Emprego da associação tetraciclina e quinino no tratamento da malária causada pelo Plasmodium falciparum. Revista da Sociedade Brasileira de Medicina Tropical 19:135-137, Jul-Set, 1986

horas. O sulfato de quinino foi iniciado juntamente com a tetraciclina, sendo fornecido por 3 dias a 51 pacientes e por 4 dias aos demais. A dose de sulfato de quinino foi de $500 \mathrm{mg}$ a cada 6 ou 8 horas, de acordo com o peso do paciente.

\section{RESULTADOS}

Todos os 110 pacientes estudados tiveram sua parasitemia negativada no período de controle póstratamento.

Setenta e cinco pacientes foram acompanhados parasitologicamente por periodo de 28 dias ou mais. A resposta ao tratamento desses 75 pacientes, avaliada de forma análoga à adotada pela Organização Mundial de Saúde para as provas "in vivo" de sensibilidade à cloroquina, foi a seguinte: $71(94,6 \%)$ pacientes tiveram resposta $S$ e $4(5,3 \%)$ resposta $R I$. As recidivas foram observadas nos dias $14,24,28$ e 37 .

Os 4 pacientes que apresentaram recidiva foram retratados com o mesmo esquema terapêutico inicial (tetraciclina e quinino). Todos foram acompanhados por 28 dias ou mais e a resposta ao segundo tratamento foi do tipo $\mathbf{S}$.

Os efeitos colaterais observados foram raros e de pequena intensidade. Além daqueles comumente observados com o uso de quinino, alguns poucos pacientes se queixaram de sensação de desconforto ou dor abdominal e diarréia que cederam espontaneamente após o término do tratamento.

Não foi observada diferença quanto à melhora clínica e ao resultado final do tratamento entre o grupo de pacientes que recebeu o sulfato de quinino por 3 dias e aquele que recebeu a droga por 4 dias.

\section{DISCUSSÃO}

A ação antimalárica de certos antibióticos é conhecida de longa data ${ }^{39}$. Esses medicamentos, entretanto, foram considerados, até recentemente, de pouca utilidade na terapêutica do impaludismo em face da existência de drogas com ação mais específica e mais imediata sobre as diferentes espécies de plasmódios.

O aparecimento de cepas de $P$. falciparum resistentes à cloroquina no início dos anos 60 e a sua disseminação pela Amazônia nos anos subseqüentes, aliados ao surgimento de cepas do parasito resistentes a outros medicamentos no decorrer da década 70 , despertaram novamente a atenção de pesquisadores para o emprego de antibióticos na terapêutica da malária.

Dentre os antibióticos com ação esquizonticida, a tetraciclina, utilizada por via oral e por periodo de 7 a 10 dias, se mostrou eficaz, mesmo quando empregada isoladamente ${ }^{3679}$. No Brasil, estudos efetuados com a minociclina confirmaram sua ação contra o $P$. falciparum $^{4}$.

A ação esquizonticida da tetraciclina, como a de todos os demais antibióticos, é lenta, e por isso recomenda-se seu uso associado a uma medicação de efeito esquizonticida rápido, para que se possa abater prontamente os sintomas e prevenir complicaçōes, sobretudo em pacientes não imunes e com parasitemia elevada 79 .

$O$ presente estudo revelou uma alta taxa de cura com o emprego de cloridrato de tetraciclina associado ao sulfato de quinino que se mostrou associação segura e isenta de efeitos colaterais relevantes. Os inconvenientes observados com o uso da tetraciclina decorreram, sobretudo, das múltiplas doses diárias do medicamento e do tempo relativamente prolongado de tratamento que dificulta a aceitação por parte de alguns pacientes.

A taxa de $5 \%$ de recidivas observadas não pode ser atribuida à verdadeira resistência do parasito à tetraciclina já que, quando retratados com o mesmo esquema terapêutico, todos os pacientes foram curados. É provável que as recidivas observadas sejam decorrentes de má absorção da droga ou do uso incorreto da medicação.

Os autores concluem que o cloridrato de tetraciclina administrado durante 7 dias e associado ao sulfato de quinino nos 3 primeiros dias de tratamento é um esquema terapêutico eficaz, barato e que, na ausência de outras alternativas, pode ser recomendado para uso em áreas não endêmicas de malária para o tratamento de infecções por $\boldsymbol{P}$. falciparum contraidas na Amazônia brasileira, resistentes à cloroquina e à associação sulfadoxina-pirimetamina.

\section{SUMMARY}

110 patients with $\mathrm{P}$. falciparum malaria and no complications from the Brazilian Amazon region were treated with the association tetracycline hydrocloride $(1 \mathrm{~g} / d$ during 7 days) and quinine sulphate (1.5 or 2 $\mathrm{g} / \mathrm{d}$ during 3 or 4 days) at the beginning of treatment. All patients were followed for at least 7 days and no blood parasites were detected ont the $7^{\text {th }}$ day. Seventyfive patients could be followed for 28 days or longer and 4 of them (5.3\%) showed parasitemia again. These results support the efficacy of the association quinine plus tetracycline in the treatment of $\mathbf{P}$. falciparum malaria infections from the Amazon region.

Key words: Chemotherapy. Quinine + Tetracycline. P. falciparum malaria.

\section{REFERÊNCIAS BIBLIOGRÁFICAS}

1. Almeida Netto JC, De Oliveira GSC, Sampaio JAA. Resistência do $P$. falciparum à associação sulfamídicos- 
Barata LCB, Boulos M, Dutra AP. Emprego da associação tetraciclina e quinino no tratamento da malária causada pelo Plasmodium falciparum. Revista da Sociedade Brasileira de Medicina Tropical 19:135-137, Jul-Set, 1986

antifólicos na região centro-oeste do Brasil. Revista de Patologia Tropical 1: 385-393, 1972.

2. Boulos M, Barata LCB. Avaliação do esquema sulfapirimetamina para malária falciparum. In: Resumos do XX Congresso da Sociedade Brasileira de Medicina Tropical, Salvador, p. 43, 1984.

3. Colwell EJ, Hickman RL, Kosakal S. Tetracycline treatment of chloroquine-resistant falciparum malaria in Thailand. Journal of the American Medical Association 220: 684-686, 1972.

4. Dourado HV. Terapêutica da malária com minociclina. Revista Brasileira de Clínica e Terapêutica 2: 10-12, 1973.

5. Moore DV, Lanier JE. Observations on two Plasmodium falciparum infections with abnormal response to chloroquine. American Journal of Tropical Medicine and Hygiene 10: 5-9, 1961.
6. Pinichpongse S, Doberstyn EB, Cullen JB, Yisunsri L, Thongsombun Y, Thimasarn K. An evaluation of five regimens for the outpatient therapy of falciparum malaria in Thailand. Bulletin of the World Health Organization 60: 907-912, 1982.

7. Reacher M, Campbell CC, Freeman J, Doberstyn EB, Brandling Bennett AD. Drug therapy for Plasmodium falciparum malaria resistant to pyrimethamine-sulfadoxine (Fansidar). Lancet 2: 1066-1068, 1981.

8. Reyes S. Infecçōes maláricas por Plasmodium falciparum resistentes ao tratamento com cloroquina. Situação no Brasil (1960-1981). Revista Brasileira de Malariologia e Doenças Tropicais 33: 109-130, 1981.

9. Rieckmann KH, Powell RD, McNamara JV, Willerson D, Kass L, Frischer H, Carson PE. Effects of tetracycline against chloroquine-resistant and chloroquine-sensitive Plasmodium falciparum. American Journal of Tropical Medicine and Hygiene 20: 811-815, 1971. 